\title{
Impact of weather factors on Mycoplasma pneumoniae pneumonia
}

\author{
D Onozuka, ${ }^{1}$ M Hashizume, ${ }^{2}$ A Hagihara ${ }^{3}$
}

\begin{abstract}
- Additional figures are published online only at http:// thorax.bmj.com/content/vol64/ issue6

${ }^{1}$ Department of Planning Information and Administration Fukuoka Institute of Health and Environmental Sciences, Dazaifu-shi, Fukuoka, Japan

${ }^{2}$ Department of International Health, Institute of Tropical Medicine, Nagasaki University, Nagasaki-shi, Nagasaki, Japan; ${ }^{3}$ Department of Health Services Management and Policy, Kyushu University Graduate School of Medicine, Higashi-ku, Fukuoka, Japan
\end{abstract}

Correspondence to: Dr D Onozuka, Department of Planning Information and Administration, Fukuoka Institute of Health and Environmental Sciences, 39 Mukaizano Dazaifu-shi, Fukuoka 818-0135 Japan; onozuka@

fihes.pref.fukuoka.jp

Received 16 November 2008 Accepted 12 March 2009

Published Online First

23 March 2009

\begin{abstract}
Background: Although multiple combinations of weather factors may contribute to an increased incidence of Mycoplasma pneumoniae pneumonia, few studies have investigated the association between weather factors and cases of $M$ pneumoniae pneumonia.
\end{abstract}

Methods: Data on cases of $M$ pneumoniae pneumonia and weather factors in Fukuoka, Japan from 1999 to 2007 were obtained and time-series analysis was used to assess the effects of weather variables on $M$ pneumoniae pneumonia cases, adjusting for confounding factors. A total of $13056 \mathrm{M}$ pneumoniae pneumonia cases were reported during the 9-year study period, of which 12234 (93.7\%) were under 15 years of age.

Results: The weekly number of $M$ pneumoniae pneumonia cases increased by $16.9 \%(95 \% \mathrm{Cl} 11.3 \%$ to $22.8 \%)$ for every $1^{\circ} \mathrm{C}$ increase in the average temperature and by $4.1 \%$ (95\% Cl $2.7 \%$ to $5.5 \%$ ) for every $1 \%$ increase in relative humidity.

Conclusions: From 1999 to 2007, cases of $M$ pneumoniae pneumonia increased significantly with increased average temperature and relative humidity in Fukuoka, Japan.

Mycoplasma pneumoniae (M pneumoniae) is a common respiratory pathogen that affects both upper and lower respiratory tract infections in all age groups. ${ }^{12}$ This agent is estimated to be responsible for $15-20 \%$ of all cases of community-acquired pneumonia (CAP) and as many as $40 \%$ of cases among children. ${ }^{2}{ }^{3}$ An estimated $30 \%$ or more of $M$ pneumoniae infections in children aged 5-15 years result in pneumonia, and as many as $18 \%$ of these cases require hospitalisation. ${ }^{45}$

$M$ pneumoniae infections can occur worldwide, with outbreaks occurring cyclically every 3-7 years. ${ }^{67}$ A study has shown that $M$ pneumoniae infection did not show seasonal variations; ${ }^{8}$ in contrast, however, recent studies have shown that $M$ pneumoniae infection peaks in winter ${ }^{9}$ or spring, ${ }^{10}$ and this aetiology should be considered first when it occurs in the summer and autumn months. ${ }^{11}$ The clear cyclical and seasonal occurrence suggests that climatic factors could play a role. Moreover, another study has shown that the most important factor explaining the variance in CAP is the direct and indirect effects of meteorological variables. ${ }^{12}$ However, few quantitative studies have investigated the impact of weather factors and variability on the incidence of $M$ pneumoniae pneumonia, allowing for the mutual confounding between weather factors and potential confounding by other seasonally varying factors. The objective of this study was to investigate the relationship between weather variations and the weekly incidence of $M$ pneumoniae pneumonia between 1999 and 2007 in Fukuoka, Japan using time-series methodology.

\section{METHODS}

\section{Data sources}

In Japan, systematic infectious disease surveillance began in 1981 under the Infectious Disease Control Law. This system, organised by the Ministry of Health and Welfare, involves about 3000 sentinel medical institutions accounting for approximately $8 \%$ of the total number of paediatric hospitals and clinics throughout the country. ${ }^{13}$ A case of $M$ pneumoniae pneumonia is defined by positive serology in combination with clinical factors. Clinical manifestations of upper respiratory tract symptoms include sore throat, hoarseness, fever, cough, headache, chills, coryza, myalgias, earache and general malaise. Infections of the lower respiratory tract generally manifest with a cough, sometimes with dyspnoea, adenopathy, wheezing and, rarely, with respiratory failure. $M$ pneumoniae respiratory tract infections are also associated with a wide range of extrapulmonary manifestations including neurological, cardiac, dermatological, musculoskeletal, haematological and gastrointestinal symptoms. ${ }^{214}$ Serological methods include passive agglutination, complement fixation and ELISA; in addition, a combination of PCR and serology is recommended for reliable diagnosis. ${ }^{15-17}$

The number of patients with $M$ pneumoniae is reported on a weekly basis from 120 sentinel medical institutions within Fukuoka Prefecture, southwest of Tokyo, Japan. The number of sentinels is based on the population of the area in which the health centre is located: a health centre with a population of $<30000$ would have one sentinel, an area with a population of 30 000-75 000 would have two, and an area with a population of $>75000$ would have three or more according to the formula ((population 75 000)/50 000). ${ }^{18}$ Clinical data are recorded and reported by sentinel volunteers to the Fukuoka Prefectural Government and the Fukuoka Institute of Health and Environmental Sciences (the municipal public health institute of Fukuoka Prefecture).

We analysed the data of 13056 cases of $M$ pneumoniae pneumonia and the meteorological data from 1999 to 2007 for Fukuoka Prefecture. We also obtained data on the daily average temperature and relative humidity in Fukuoka Prefecture from the Japan Meteorological Agency. The weekly means for average temperature and relative humidity were calculated from the daily records. 


\section{Statistical analysis}

The relationship between the number of weekly $M$ pneumoniae pneumonia cases and the temperature or humidity was analysed using generalised linear Poisson models allowing for overdispersion. ${ }^{19}$ We used mean temperature and humidity as they were better predictors of temperature and humidity relationships than either maximum or minimum temperature and humidity by the maximum likelihoods for the models. To account for the seasonality of $M$ pneumoniae pneumonia cases not directly due to weather factors, Fourier terms up to the sixth harmonic were included in the model. Fourier terms can capture repeated periodic (eg, seasonal) patterns comprising a combination of pairs of sine and cosine terms (harmonics) of varying wavelength. ${ }^{20}$ Indicator variables for each year were incorporated into the model to allow for long-term trends and other variations occurring over the 9 years of the study. To allow for autocorrelations, an autoregressive term at order 1 was incorporated into the models. ${ }^{21}$ Plots of model residuals, predicted and observed time series plots and partial autocorrelation function of the residuals (see fig S1 in online supplement) suggested that this was an adequate amount of adjustment for seasonal trends.

\section{Temperature models}

Based on the results of exploratory analyses, existing literature $^{22}{ }^{23}$ and considerations of interpretational difficulty with very long lag periods, we considered lag periods (delays in effect) of up to 8 weeks for the influence of temperature on the number of $M$ pneumoniae pneumonia cases. In the initial analyses designed to identify the broad shape of any association, we fitted a natural cubic spline ( $3 \mathrm{df}$ ) to the average over lag periods of $0-8$ weeks. ${ }^{24}$ We also included humidity as a natural cubic spline $(3 \mathrm{df})$ in the model to control for the effects of confounding, with a lag of 0-8 weeks.

The choice of model (linear or threshold) was based on comparing the deviance of the models derived from likelihood ratio tests. ${ }^{25}$ The model with the smallest deviance was preferred. When a difference in values of deviance between linear and the best-fit threshold models was less than $3.84\left(\chi^{2}\right.$ value for one degree of freedom at the $p=0.05$ level), the linear model was chosen for simplicity. Likelihood profiles suggested that the deviance between the linear and threshold models changed little; we therefore assumed that linear models without a threshold were appropriate for assessing the effects of weather variability on cases of $M$ pneumoniae pneumonia.

Using the simple linear model, we then examined lag effects in greater detail by fitting linear unconstrained distributed lag models comprising temperature terms at each lag period that could be as long as 8 weeks.

Table 1 Characteristics of the weekly number of cases of Mycoplasma pneumoniae pneumonia and meteorological data in Fukuoka, Japan, 1999-2007

\begin{tabular}{|c|c|c|c|c|c|}
\hline \multirow[b]{2}{*}{ Variable (unit) } & \multirow[b]{2}{*}{ Minimum } & \multicolumn{3}{|c|}{ Percentile } & \multirow[b]{2}{*}{ Maximum } \\
\hline & & 25 & 50 & 75 & \\
\hline $\begin{array}{l}\text { Number of } M \text { pneumoniae } \\
\text { pneumonia cases }\end{array}$ & 4 & 15 & 24 & 36 & 96 \\
\hline Average temperature $\left({ }^{\circ} \mathrm{C}\right)$ & 2.9 & 10.6 & 17.5 & 24.4 & 30.5 \\
\hline Average humidity (\%) & 43.9 & 60.1 & 65.5 & 70.7 & 83.9 \\
\hline
\end{tabular}
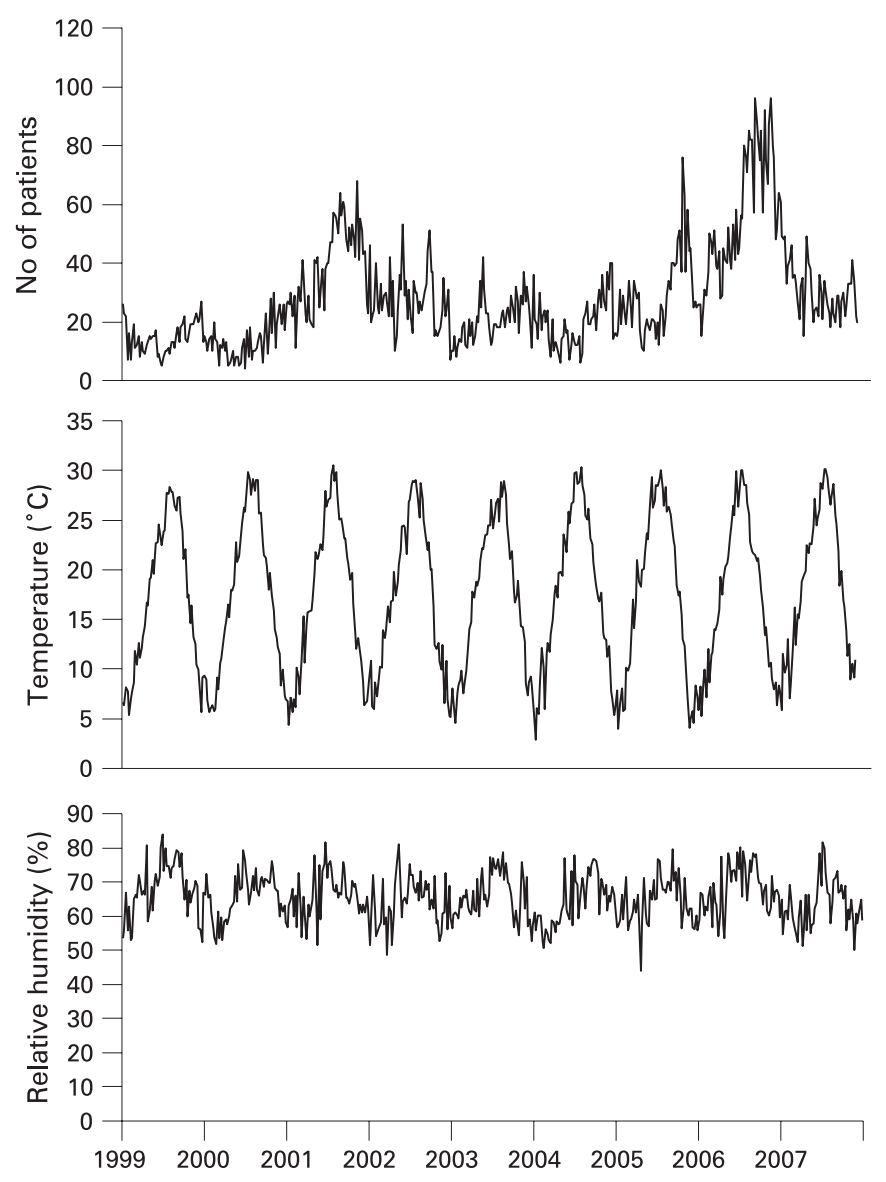

Figure 1 Seasonal variations in the weekly number of cases of Mycoplasma pneumoniae pneumonia, temperature and humidity in Fukuoka, Japan, 1999-2007.

\section{Humidity models}

Humidity was analysed because we hypothesised that, together with temperature, humidity is a potential causal factor of a multiple factor pathway for the incidence of $M$ pneumoniae pneumonia cases. Specifically, we fitted a natural cubic spline ( $3 \mathrm{df}$ ) to the average humidity over lag periods of $0-8$ weeks and incorporated this into a model comprising the same confounders included in the temperature model. As in the temperature models, the lag period was set at 0-8 weeks.

Because the plots of the smoothed relationships with humidity suggested a broadly linear positive relationship and the deviance between the linear and threshold models changed little, we fitted a linear model to estimate the effect (slope). ${ }^{25}$ With the simple linear model, we examined lag effects in more detail by fitting linear unconstrained distributed lag models comprising humidity terms at each lag period that could be as long as 8 weeks.

We then examined whether the association still held if the two epidemics in 2001 and 2006 were removed from the analysis, because the large number of cases during two epidemics coinciding with high temperature and humidity may have skewed the data. In addition, to investigate whether the results were sensitive to the levels of control for seasonal patterns, the sensitivity analyses were conducted using different degrees of seasonal control ( 3 and 12 harmonics). All statistical analyses were carried out using Stata 10.1 (Stata Corporation, College Station, Texas, USA). 


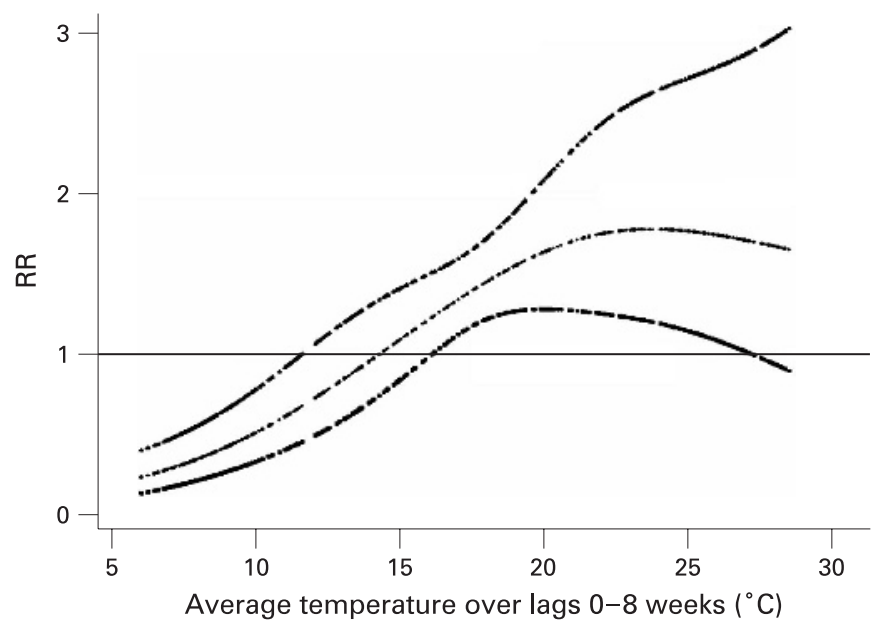

Figure 2 Relationship adjusted for relative humidity, seasonal variations and between-year variations between relative risk (RR) of Mycoplasma pneumoniae pneumonia (scaled to the mean weekly number of $M$ pneumoniae pneumonia cases) and temperature over lag periods of 0-8 weeks (shown as a 3 df natural cubic spline). The centre line shows the estimated spline curve and the upper and lower lines represent the $95 \%$ confidence limits.

\section{RESULTS}

A total of 13056 cases of $M$ pneumoniae pneumonia were analysed from 1999 to 2007, of which 211 (1.6\%) were infants aged $<12$ months, $1966(15.1 \%)$ were $1-2$ years of age, 3081 (23.6\%) were 3-4 years of age, $5346(40.9 \%)$ were $5-9$ years of age, $1630(12.5 \%)$ were $10-14$ years of age and $822(6.3 \%)$ were aged $\geqslant 15$ years. Descriptive statistics for the number of patients and weather variables are shown in table 1 . Analysis of the weekly reported cases of $M$ pneumoniae pneumonia showed that the seasonal peak in cases was not identical from year to year (fig 1). The data were analysed to look for synergy between temperature and humidity.

\section{Relationship with temperature}

The relationships between the relative risk of $M$ pneumoniae pneumonia cases and temperature are shown in fig 2 . In the crude relationship, the potential risk of $M$ pneumoniae pneumonia increased as temperature increased from the lowest temperatures (see fig S2 in online supplement). A significant positive relationship was found between the relative risk of $M$ pneumoniae pneumonia and the presence of higher temperatures during a lag of $0-8$ weeks after adjusting for seasonal, betweenyear and humidity variations (fig 2). For a $1^{\circ} \mathrm{C}$ increase, the number of cases of $M$ pneumoniae pneumonia increased by $16.9 \%$ (95\% CI $11.3 \%$ to $22.8 \%$ ) and the temperature effect was significant for lag periods of 7 weeks using the distributed lag model. Little effect was observed for the other lag periods (fig 3).

\section{Relationship with humidity}

The relationship between the relative risk of $M$ pneumoniae pneumonia and humidity is shown in fig 4. In the crude relationship, the potential risk of $M$ pneumoniae pneumonia increased as relative humidity increased from the lowest relative humidity (see fig S3 in online supplement). After adjusting for seasonal, between-year and temperature variations, we observed a significant increase in the number of cases of $M$ pneumoniae pneumonia with a $1 \%$ increase in relative humidity for lag periods between 0 and 8 weeks, as indicated by the positive

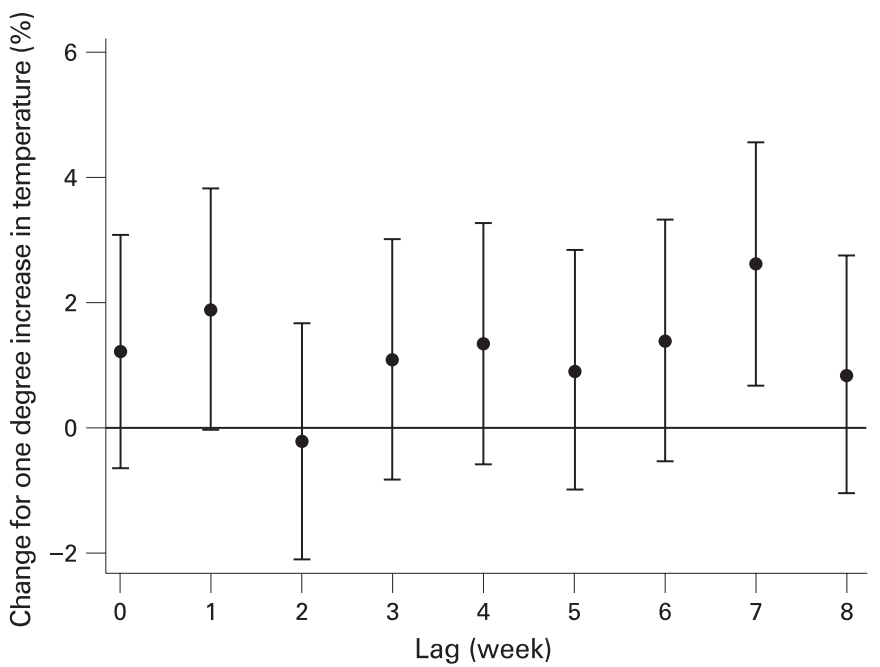

Figure 3 Percentage change (and $95 \% \mathrm{Cl}$ ) in the number of cases of Mycoplasma pneumoniae pneumonia per $1{ }^{\circ} \mathrm{C}$ increase in temperature at each lag (unconstrained distributed lag models).

linear slope with high humidity (fig 4). For a $1 \%$ increase in humidity, the number of $M$ pneumoniae pneumonia cases increased by $4.1 \%$ (95\% CI $2.7 \%$ to $5.5 \%)$. The effect of humidity was significant at the lag periods of $3,4,5,6,7$ and 8 weeks using the distributed lag model. Little effect was observed for the other lag periods (fig 5).

As the result of analysis without the two epidemics in 2001 and 2006, the weekly number of cases of $M$ pneumoniae pneumonia increased by $8.4 \%$ (95\% CI $2.3 \%$ to $14.9 \%$ ) for every $1{ }^{\circ} \mathrm{C}$ increase in the average temperature and by $4.1 \%(95 \%$ CI $2.6 \%$ to $5.7 \%$ ) for every $1 \%$ increase in relative humidity. In sensitivity analyses, when the degree of seasonal control was halved ( 3 harmonics) or doubled (12 harmonics), the estimates of the effect of temperature and humidity changed little.

\section{DISCUSSION}

Several notable points are concluded from our findings. Most importantly, our results suggest that, after adjusting for potential confounding by temperature (only in the humidity models), humidity (only in the temperature models), seasonal patterns and between-year variations, there was evidence for an increase in the number of $M$ pneumoniae pneumonia cases with either an increase in temperature or relative humidity. Moreover, these positive associations still held after removing the two strong epidemics in 2001 and 2006. A laboratory-based study suggested that the survival of airborne $M$ pneumoniae was found to be a function of both temperature and relative humidity, however, the temperature response was mediated by humidity in that the effects of temperature could only be observed if some water vapour was present. ${ }^{26}$ In addition, at all temperatures, survival of $M$ pneumoniae in aerosols was found to be best at extremes of relative humidity and the effects of temperature were such that, irrespective of relative humidity, an increase in temperature resulted in a decreased airborne survival time. ${ }^{26}$ However, the result of an epidemiological longitudinal study suggested that the survival and spread of $M$ pneumoniae were highly favoured during the spring and early autumn seasons and there might be a positive relationship between $M$ pneumoniae infections and temperature, which was only indirectly inferred from interannual observations or seasonal variations. ${ }^{27}$ The positive relationship between $M$ 


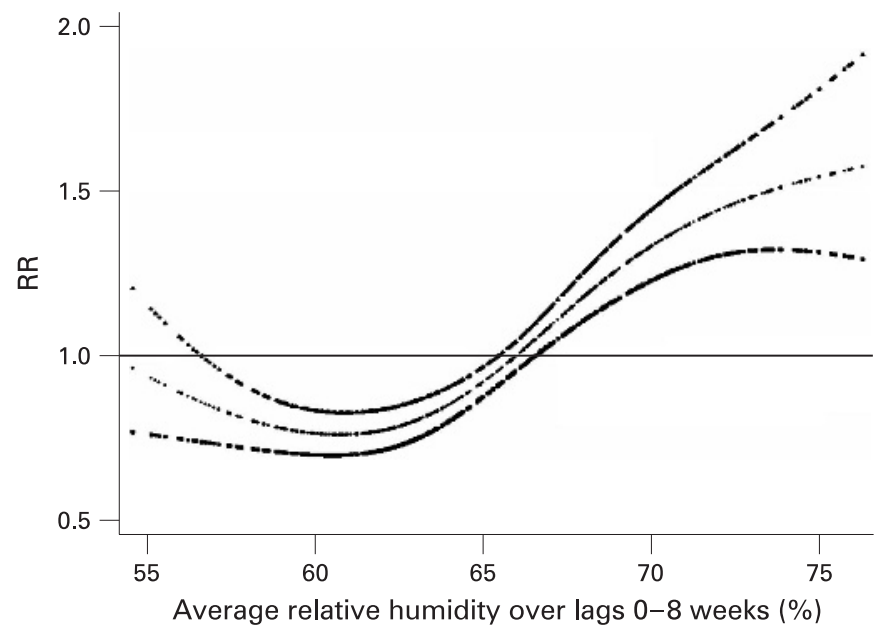

Figure 4 Relationship adjusted for temperature, seasonal variations and between-year variations between relative risk (RR) of Mycoplasma pneumoniae pneumonia (scaled to the mean weekly number of $M$ pneumoniae pneumonia cases) and relative humidity over lag periods of 0-8 weeks (shown as a $3 \mathrm{df}$ natural cubic spline). The centre line shows the estimated spline curve and the upper and lower lines represent the $95 \%$ confidence limits.

pneumoniae pneumonia cases and increased temperature in this study is therefore broadly consistent with the previous finding.

Our study also found that the number of cases of $M$ pneumoniae pneumonia was increased with an increase in relative humidity independent of the ambient temperature. With respect to an association between the prevalence of $M$ pneumoniae pneumonia cases and humidity, a laboratory-based study has suggested that survival of airborne $M$ pneumoniae was found to be best at $27^{\circ} \mathrm{C}$ and $<25 \%$ or $90 \%$ relative humidity, while the most lethal relative humidity levels were at $60 \%$ and $80 \%{ }^{28}$ Another laboratory-based study indicated that the biological stability of airborne $M$ pneumoniae might be easily modified by a sudden change in the relative humidity, such as occurs in the natural atmosphere. ${ }^{29}$ On the other hand, some epidemiological studies have indicated that there was little relationship between $M$ pneumoniae infections and relative humidity. ${ }^{10}{ }^{30}$ However, the former findings were studied at variable relative humidity with a controlled temperature and the latter were studied by descriptive analysis. The discrepancy could be due to the effects of seasonally varying factors and mutual confounding between weather factors; these were controlled in the current study, but not in the previous one. In any case, our combined temperature and humidity results demonstrate the importance of weather factors on the prevalence of $M$ pneumoniae pneumonia infections.

Using the distributed lag model, the temperature effects appeared immediately at shorter lag periods (0-1 weeks) and were generally positive at later lags. In contrast, the humidity effects seemed to be delayed by a week or two, and were significantly positive throughout the lag periods around 3-8 weeks. These results may be related to the fact that $M$ pneumoniae has a long incubation period of the organism and late detection of outbreaks. ${ }^{31}$ Because of these characteristics of $M$ pneumoniae, public health officials and healthcare providers struggle-often with little success-to detect $M$ pneumoniae pneumonia infections. This may also indicate that more precise modelling of any lag effects of temperature and humidity on the risk of disease and further discussion of disease-specific issues is needed.

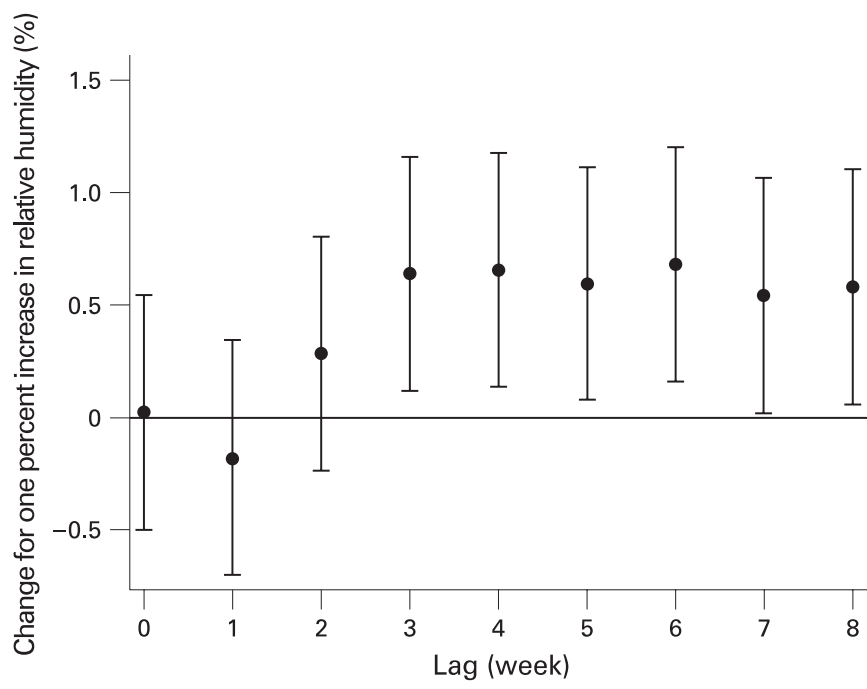

Figure 5 Percentage change (and 95\% CI) in the number of cases of Mycoplasma pneumoniae pneumonia per $1 \%$ increase in humidity at each lag (unconstrained distributed lag models).

There may be concerns that not all cases in the community are represented in surveillance data. This under-reporting can occur anywhere in the reporting chain, from the initial tendency of a patient to seek health care to the recording of the case in the disease registry. M pneumoniae infections commonly occur in closed or semi-closed communities, ${ }^{32}{ }^{33}$ and most people with $M$ pneumoniae infections have relatively mild disease symptoms ${ }^{34}$ such as a cough or sore throat, or no symptoms at all. ${ }^{35}$ Thus, containing $M$ pneumoniae infections is difficult. However, we find no reason to believe this would result in substantial bias because the degree of under-reporting is not likely to vary over time. Another concern may be related to the fact that sentinel medical institutions were recruited on a voluntary basis, but this does not pose a threat to the validity of the comparisons over time, which is the subject of this study.

The short-term associations reported here cannot be directly extrapolated to changes in climate over decades. While it remains uncertain how increases in warmer temperatures could affect $M$ pneumoniae infections, the results of this study suggest that heat-related $M$ pneumoniae pneumonia cases are likely to increase with climate change in Fukuoka, Japan.

Finally, we would like to refer to the practical implications of the present findings. Understanding the effects of weather variability on the epidemiology of infectious diseases is important for planning health services. These observed associations between variability in the weather and adverse health effects could represent a possible model or analogue for the impact on health of future climate changes. Health services may need to prepare for the effects of climate change on the epidemiology of $M$ pneumoniae pneumonia through the implementation of preventive public health interventions. Such interventions might include alerting health workers, intensive community-based campaigns, weather forecasting for early warning and planning additional control programmes for $M$ pneumoniae pneumonia. In accordance with these interventions, we might have a change in behaviour, such as people coming into closer contact with each other and thereby increasing the chance of person-to-person transmission.

In conclusion, this study found quantitative evidence that the number of cases of $M$ pneumoniae pneumonia increased with higher temperature and relative humidity in the weeks 
preceding disease onset for a large number of cases over a 9-year period.

Acknowledgements: The authors thank the Fukuoka Prefectural Government, Department of Public Health and Medical Affairs, Division of Public Health.

Funding: The study was supported in part by a Grant-in-Aid for Scientific Research from the Ministry of Health, Labour and Welfare, Japan.

Competing interests: None.

\section{REFERENCES}

1. Esposito S, Cavagna R, Bosis S, et al. Emerging role of Mycoplasma pneumoniae in children with acute pharyngitis. Eur J Clin Microbiol Infect Dis 2002:21:607-10.

2. Waites KB, Talkington DF. Mycoplasma pneumoniae and its role as a human pathogen. Clin Microbiol Rev 2004;17:697-728.

3. Marston BJ, Plouffe JF, File TM Jr, et al. Incidence of community-acquired pneumonia requiring hospitalization. Results of a population-based active surveillance Study in Ohio. The Community-Based Pneumonia Incidence Study Group. Arch Intern Med 1997:157:1709-18.

4. Korppi M, Heiskanen-Kosma T, Kleemola M. Incidence of community-acquired pneumonia in children caused by Mycoplasma pneumoniae: serological results of a prospective, population-based study in primary health care. Respirology 2004;9:109-14.

5. Hammerschlag MR. Mycoplasma pneumoniae infections. Curr Opin Infect Dis 2001;:14:181-6.

6. Lind K, Benzon MW, Jensen JS, et al. A seroepidemiological study of Mycoplasma pneumoniae infections in Denmark over the 50-year period 1946-1995. Eur J Epidemiol 1997:13:581-6.

7. Layani-Milon MP, Gras I, Valette M, et al. Incidence of upper respiratory tract Mycoplasma pneumoniae infections among outpatients in Rhone-Alpes, France, during five successive winter periods. J Clin Microbiol 1999;37:1721-6.

8. Foy HM, Kenny GE, Cooney MK, et al. Long-term epidemiology of infections with Mycoplasma pneumoniae. J Infect Dis 1979:139:681-7.

9. Sidal M, Kilic A, Unuvar E, et al. Frequency of Chlamydia pneumoniae and Mycoplasma pneumoniae infections in children. J Trop Pediatr 2007;53:225-31.

10. Lieberman D, Lieberman D, Porath A. Seasonal variation in community-acquired pneumonia. Eur Respir J 1996;9:2630-4.

11. Mansel JK, Rosenow EC 3rd, Smith TF, et al. Mycoplasma pneumoniae pneumonia. Chest 1989;95:639-46.

12. Lieberman D, Lieberman D, Friger MD. Seasonal variation in hospital admissions for community-acquired pneumonia: a 5-year study. J Infect 1999;39:134-40.

13. Hashimoto S, Murakami Y, Taniguchi K, et al. Detection of epidemics in their early stage through infectious disease surveillance. Int J Epidemiol 2000;29:905-10.

14. Tsiodras S, Kelesidis I, Kelesidis T, et al. Central nervous system manifestations of Mycoplasma pneumoniae infections. J Infect 2005;51:343-54.

15. Dorigo-Zetsma JW, Zaat SA, Wertheim-van Dillen PM, et al. Comparison of PCR, culture, and serological tests for diagnosis of Mycoplasma pneumoniae respiratory tract infection in children. J Clin Microbiol 1999;37:14-7.

16. Templeton KE, Scheltinga SA, Graffelman AW, et al. Comparison and evaluation of real-time PCR, real-time nucleic acid sequence-based amplification, conventional PCR, and serology for diagnosis of Mycoplasma pneumoniae. J Clin Microbiol 2003:41:4366-71

17. Welti M, Jaton $\mathrm{K}$, Altwegg $\mathbf{M}$, et al. Development of a multiplex real-time quantitative PCR assay to detect Chlamydia pneumoniae, Legionella pneumophila and Mycoplasma pneumoniae in respiratory tract secretions. Diagn Microbiol Infect Dis 2003:45:85-95.

18. Murakami Y, Hashimoto $S$, Taniguchi $K$, et al. Distributions of the numbers of monitoring stations in the surveillance of infectious diseases in Japan. Nippon Koshu Eisei Zasshi 1999:46:1060-7.

19. McCullagh P, Nelder J. Generalized linear models. London: Chapman and Hall, 1989

20. Stolwijk AM, Straatman H, Zielhuis GA. Studying seasonality by using sine and cosine functions in regression analysis. J Epidemiol Community Health 1999:53:235-8.

21. Brumback B, Burge HA, Neas LM, et al. Transitional regression models, with application to environmental time series. J Am Stat Assoc 2000;95:16-27.

22. Hashizume M, Armstrong B, Hajat $\mathrm{S}$, et al. Association between climate variabilit/ and hospital visits for non-cholera diarrhoea in Bangladesh: effects and vulnerable groups. Int J Epidemiol 2007;36:1030-7.

23. Hashizume M, Armstrong B, Hajat $\mathrm{S}$, et al. The effect of rainfall on the incidence of cholera in Bangladesh. Epidemiology 2008:19:103-10.

24. Durrleman S, Simon R. Flexible regression models with cubic splines. Stat Med 1989:8:551-61.

25. Daniels MJ, Dominici F, Samet JM, et al. Estimating particulate matter-mortality dose-response curves and threshold levels: an analysis of daily time-series for the 20 largest US cities. Am J Epidemiol 2000;152:397-406.

26. Wright DN, Bailey GD, Goldberg LJ. Effect of temperature on survival of airborne Mycoplasma pneumoniae. J Bacteriol 1969;99:491-5

27. Edwards EA, Crawford YE, Pierce WE, et al. A longitudinal study of Mycoplasma pneumoniae: infections in Navy recruits by isolation and seroepidemiology Am J Epidemiol 1976;104:556-62.

28. Wright DN, Bailey GD, Hatch MT. Role of relative humidity in the survival of airborne Mycoplasma pneumoniae. J Bacteriol 1968;96:970-4.

29. Hatch MT, Wright DN, Bailey GD. Response of airborne Mycoplasma pneumoniae to abrupt changes in relative humidity. Appl Microbiol 1970;19:232-8.

30. Beskow $\mathbf{P}$, Norqvist M, Wallgren P. Relationships between selected climatic factors in fattening units and their influence on the development of respiratory diseases in swine. Acta Vet Scand 1998;39:49-60.

31. Ancel Meyers L, Newman ME, Martin M, et al. Applying network theory to epidemics: control measures for Mycoplasma pneumoniae outbreaks. Emerg Infect Dis 2003:9:204-10.

32. Feikin DR, Moroney JF, Talkington DF, et al. An outbreak of acute respiratory disease caused by Mycoplasma pneumoniae and adenovirus at a federal service training academy: new implications from an old scenario. Clin Infect Dis 1999;29:1545-50

33. Klausner JD, Passaro D, Rosenberg J, et al. Enhanced control of an outbreak of Mycoplasma pneumoniae pneumonia with azithromycin prophylaxis. J Infect Dis 1998;177:161-6.

34. Foy HM, Kenny GE, McMahan R, et al. Mycoplasma pneumoniae in the community Am J Epidemiol 1971;93:55-67.

35. Foy HM, Grayston JT, Kenny GE, et al. Epidemiology of Mycoplasma pneumoniae infection in families. JAMA 1966:197:859-66.

\section{Quality \& Safety in Health Care}

Quality \& Safety in Health Care is a leading international peer-review journal in the growing area of quality and safety improvement. It provides essential information for those wanting to reduce harm and improve patient safety and the quality of care. The journal reports and reflects research, improvement initiatives and viewpoints and other discursive papers relevant to these crucial aims with contributions from researchers, clinical professionals and managers and experts in organisational development and behaviour.

qshc.bmj.com 